\title{
IMPACTOS DA MUDANÇA DE VEGETAÇÃO LOCAL NO APORTE DE MATÉRIA ORGÂNICA PARA UM ESTUÁRIO TROPICAL PRESERVADO (ESTUÁRIO DO RIO ITAPICURU - BA)
}

\author{
Lucas M. Guimarães ${ }^{a}$, Tiago de Abreu C. Limaa, Elvis Joacir De Françab ${ }^{\mathrm{b}}$ Gilberto N. de Arruda ${ }^{\mathrm{b}}$, José Roberto B. de Souza \\ e Ana Cecília R. de Albergaria-Barbosa $a^{\mathrm{a}, *, \mathbb{1}}$ \\ aDepartamento de Oceanografia, Instituto de Geociências, Universidade Federal da Bahia, 40170-115 Salvador - BA, Brasil \\ ${ }^{b}$ Centro Regional de Ciências Nucleares do Nordeste, Comissão Nacional de Energia Nuclear, 50740-545 Recife - PE, Brasil \\ ${ }^{\mathrm{c}}$ Departamento de Física da Terra e Meio Ambiente, Instituto de Física, Universidade Federal da Bahia, 40170-115 Salvador - BA, \\ Brasil
}

Recebido em 08/04/2019; aceito em 09/05/2019; publicado na web em 23/05/2019

\begin{abstract}
IMPACTS OF LOCAL VEGETATION CHANGES IN THE ORGANIC MATTERS INPUT FOR A PRESERVED TROPICAL ESTUARY (ITAPICURU - BA RIVER ESTUARY). Estuaries are major areas of organic matter (OM) deposition, playing an important role in the global carbon cycle. The Itapicuru's river estuary (IRE) is in the North Coast of Bahia (Brazil), in an Environmental Protected Area. Although it presents preserved conditions, some environmental changes caused by human activities can be observed. This study evaluated the anthropogenic impacts importance in concentrations/sources of OM deposited in IRE. $n$-Alkanes and stable isotopes $\left(\delta^{13} \mathrm{C}\right.$ and $\left.\delta^{15} \mathrm{~N}\right)$ were analyzed in a sediment core collected in the studied region. Total $n$-alkanes $\left(n\right.$ - $\mathrm{C}_{15}$ to $n-\mathrm{C}_{35}$ ), TOC and TN concentrations varied from 1,333 to $7,384 \mathrm{ng} \mathrm{g}^{-1}, 0.65 \%$ to $2.51 \%$ and $0.07 \%$ to $0.13 \%$, respectively. Values of $\delta^{13} \mathrm{C}$ and $\delta^{15} \mathrm{~N}$ ranged from $-28.8 \%$ to $-24.3 \%$ o, $-6.23 \%$ o to $1.87 \%$, respectively. By the means of the isotopic composition and the selected $n$-alkanes diagnostic ratios values (Productivity aquatic index; Carbon Preference Index; Average Chain Length and Alkane index), the main OM sources for IRE were determined as $\mathrm{C}_{3}$ terrestrial plants. This area is covered by mangrove/riparian vegetation, which mainly species have this photosynthetic pathway. Temporal changes in deposited OM occurred due natural and anthropogenic processes, such urban growth in the IRE upstream region.
\end{abstract}

Keywords: $n$-alkanes; stable isotopes; organic matter; sediment core; estuary.

\section{INTRODUÇÃO}

As zonas costeiras são importantes compartimentos dentro do ciclo global do carbono, pois cerca de $80 \%$ da matéria orgânica (M.O.) global é depositada nessas regiões. ${ }^{1}$ A M.O. natural encontrada em sistemas estuarinos é uma mistura complexa de macro e micromoléculas de massas moleculares não homogêneas que podem ser encontradas adsorvidas nos sedimentos. ${ }^{1-4}$ Suas principais fontes naturais para ambientes costeiros podem ser classificadas como: autóctones (derivadas de bactérias e macrófitas marinhas, bem como aquelas provindas da produção fitoplanctônica e algal) e alóctones (derivadas de ecossistemas terrestres circundantes, como manguezal adjacente a estuários ou vegetação provinda ao longo do curso de rios). ${ }^{4-6}$ Conhecer a distribuição, as fontes e a composição da M.O. natural depositada em sistemas estuarinos é essencial para entender os mecanismos que controlam sua distribuição. ${ }^{7}$ Além disso, a compreensão da M.O. sedimentar é importante, uma vez que ela é componente dos ciclos biogeoquímicos globais. ${ }^{8}$

Os estudos de caracterização da M.O. baseiam-se, geralmente, na análise isotópica do carbono orgânico e do nitrogênio. ${ }^{9-12}$ Esta avaliação é possível devido ao fato de que diferentes produtores primários produzem M.O. com razões isotópicas características. ${ }^{13}$ Essa distinção da M.O. também é feita através de indicadores geoquímicos orgânicos no nível molecular, comumente chamados de marcadores orgânicos moleculares. ${ }^{2,14-17}$ Estes compostos são utilizados para os estudos ambientais por serem lipofílicos/hidrofóbicos, resistentes à biodegradação, apresentarem alta estabilidade química, e por terem especificidade de fonte. ${ }^{18,19}$ Dentre os marcadores geoquímicos usados para caracterização da matéria orgânica, destacam-se os

*e-mail: cecilia.albergaria@ufba.br $n$-alcanos. ${ }^{20-23}$ Estes compostos são sintetizados por plantas terrestres (com padrão fotossintético do tipo $\mathrm{C}_{3}$ e $\mathrm{C}_{4}$ ), macrófitas (emersas, flutuantes ou submersas) e pelo fitoplâncton. ${ }^{20,21,24}$ Esses organismos biossintetizam os $n$-alcanos através da descarboxilação de ácidos graxos, contendo principalmente número ímpar de carbonos em suas cadeias. ${ }^{25}$ Organismos fitoplanctônicos, por estarem em meio aquático, normalmente sintetizam compostos com cadeias menores que 20 átomos de carbono $\left(n-\mathrm{C}_{15}, n-\mathrm{C}_{17}\right.$ e $\left.n-\mathrm{C}_{19}\right)$. As macrófitas sintetizam preferencialmente compostos com 23 a 25 átomos de carbono. ${ }^{20}$ Plantas superiores sintetizam $n$-alcanos com cadeias acima de 27 átomos de carbono, principalmente entre $n-\mathrm{C}_{29}$ e $n-\mathrm{C}_{33}$. O padrão fotossintético do tipo $\mathrm{C}_{3}$ ou $\mathrm{C}_{4}$ das plantas superiores também influenciará na biossíntese dos $n$-alcanos. ${ }^{21}$

O estuário do rio Itapicuru está localizado na cidade de Conde (BA), no nordeste do Brasil. Ele está inserido na unidade de conservação da Área de Proteção Ambiental do Litoral Norte da Bahia (APA/LN), que foi criada como um instrumento mitigador do avanço populacional na região, devido à implantação da rodovia BA-099 (Linha Verde). ${ }^{26}$ A vegetação característica que ocorre ao longo do estuário do rio Itapicuru é a Mata Atlântica. Apesar de ser considerada bem preservada e desenvolvida, a partir da década de 1970, ela foi trocada por florestas comerciais de pinus e eucaliptos para produção de celulose e carvão vegetal e pela agropecuária. ${ }^{27-29}$ Nas últimas décadas, houve também a troca da Mata Atlântica pelo cultivo de coqueiros. Atualmente, o município de Conde, localizado ao lado do estuário do rio Itapicuru, é um dos principais produtores de coco do Brasil. ${ }^{29}$ Outras atividades locais, como agricultura familiar, pesca, pecuária extensiva e turismo, também ocasionam o desmatamento de matas ciliares na região. ${ }^{27}$ Com a implantação da BA-099 (década de 90), o litoral norte, incluindo a cidade de Conde, começou a sofrer um processo de especulação imobiliária, com apropriação, 
concentração e valorização do espaço. ${ }^{30}$ Outros conflitos ambientais que o litoral norte da Bahia sofre são: ocupação desordenada do solo, falta de saneamento básico, impactos ambientais causados por áreas de plantação de pinus e eucalipto, intensificação indiscriminada da pecuária e pesca predatória, degradação dos manguezais e o turismo predatório nos distritos litorâneos. ${ }^{26}$ Todas estas atividades conjuntas têm eliminado parte da cobertura vegetal típica da região. ${ }^{31}$ No estuário do rio Itapicuru estão localizadas as unidades ambientais mais frágeis e relevantes do Litoral Norte baiano, como o maior manguezal da APA/LN e suas restingas. ${ }^{32}$

O principal objetivo deste estudo foi avaliar as mudanças temporais no aporte e composição da matéria orgânica depositada no estuário do rio Itapicuru (BA), associando-as com a troca da vegetação nativa e com o desenvolvimento urbano da região. Esse objetivo irá elucidar como que as atividades antropogênicas e eventos ambientais influenciam no tipo/quantidade de matéria orgânica depositada em estuários tropicais relativamente preservados. Além disso, informações disponíveis sobre estuários localizados no nordeste do Brasil são limitados, sendo importante o melhor entendimento sobre o funcionamento geoquímico destes sistemas.

\section{MATERIAIS E MÉTODOS}

\section{Área de estudo}

O estuário do rio Itapicuru está sob domínio municipal da cidade de Conde, Litoral Norte do Estado da Bahia, entre as latitudes $11^{\circ} 44^{\prime}-11^{\circ} 49^{\prime} \mathrm{S}$ e as longitudes $37^{\circ} 30^{\prime}-37^{\circ} 35^{\prime}$ O (Figura 1). A descarga fluvial média do rio Itapicuru é de $40,5 \mathrm{~m}^{3} \mathrm{~s}^{-1} \cdot{ }^{33}$ Seu regime de maré é semidiurna, com amplitudes máximas variando entre 2,0 e 2,5 m. ${ }^{34,35} \mathrm{~A}$ ação das ondas provenientes do sudeste e sul-sudeste, seguida das marés, são os principais agentes hidrodinâmicos da região. ${ }^{29,36}$ Esses são os principais agentes controladores da distribuição sedimentar no canal estuarino e, por sua vez, da tendência de deriva litorânea local para nordeste. ${ }^{29,37,38}$ As temperaturas médias para a região são elevadas (entre $24^{\circ}$ e $26^{\circ} \mathrm{C}$ ), com amplitudes térmicas variando entre $3-6{ }^{\circ} \mathrm{C}$, e altos índices pluviométricos anuais (entre $1000 \mathrm{~mm}$ à $1400 \mathrm{~mm})$. $^{39-41}$

O munícipio de Conde, região litorânea do rio Itapicuru, é composto por vegetação praial, zonas úmidas, manguezal, bem como a restinga em moitas e matas de restinga, sendo essas duas últimas as predominantes. ${ }^{42}$ Minervino-Netto ${ }^{35}$ destaca ainda que, na localidade de Sítio do Conde, no município de Conde, ocorre a mais expressiva floresta paludosa (floresta de brejo) do Estado da Bahia caracterizada como zona úmida. Estudos realizados por Menezes et al. ${ }^{42}$ na região da cidade de Conde, identificaram um total de 142 espécies de vegetação pertencentes a 67 famílias botânicas, sendo a Cyperaceae a de maior riqueza específica, com 16 espécies. Porém, as espécies da família Polygonnaceae e Myrtaceae dominam na região de Conde. A Rhizophora mangle é a espécie que predomina neste ecossistema de mangue. Estas florestas são representativas do clima tropical quente, úmido e subúmido, todas típicas do bioma Mata Atlântica. ${ }^{42}$

\section{Amostragem}

Em janeiro de 2017, um testemunho sedimentar foi coletado ao longo da margem do estuário do rio Itapicuru (BA) nas coordenadas $11^{\circ} 45^{\prime} 16^{\prime \prime} \mathrm{S}$ e $37^{\circ} 31^{\prime} 17^{\prime \prime} \mathrm{O}$ (Figura 1). O testemunho foi mantido a $-20{ }^{\circ} \mathrm{C}$ e posteriormente seccionado em sub-amostras de $2 \mathrm{~cm}$, sendo estas acondicionadas em frascos de vidro calcinados $\left(400{ }^{\circ} \mathrm{C}\right.$ por $4 \mathrm{~h}$ ) liofilizadas.

\section{Reagentes}

$\mathrm{O}$ ácido clorídrico $(\mathrm{HCl}, \mathrm{PA})$ e o peróxido de hidrogênio $\left(\mathrm{H}_{2} \mathrm{O}_{2}\right)$ usados na realização do presente estudo foram obtidos pela Merck. Os solventes utilizados foram: o $n$-hexano (HEX, com $95 \%$ de grau de pureza) e o diclorometano (DCM, com 99,9\% de grau de pureza), ambos fornecidos pela JT Baker. A sílica $\left(\mathrm{SiO}_{2}\right.$ - sílica gel 60 x 0,063 x 0,200 mm, Merck) e o sulfato de sódio $\left(\mathrm{Na}_{2} \mathrm{SO}_{4}\right.$,
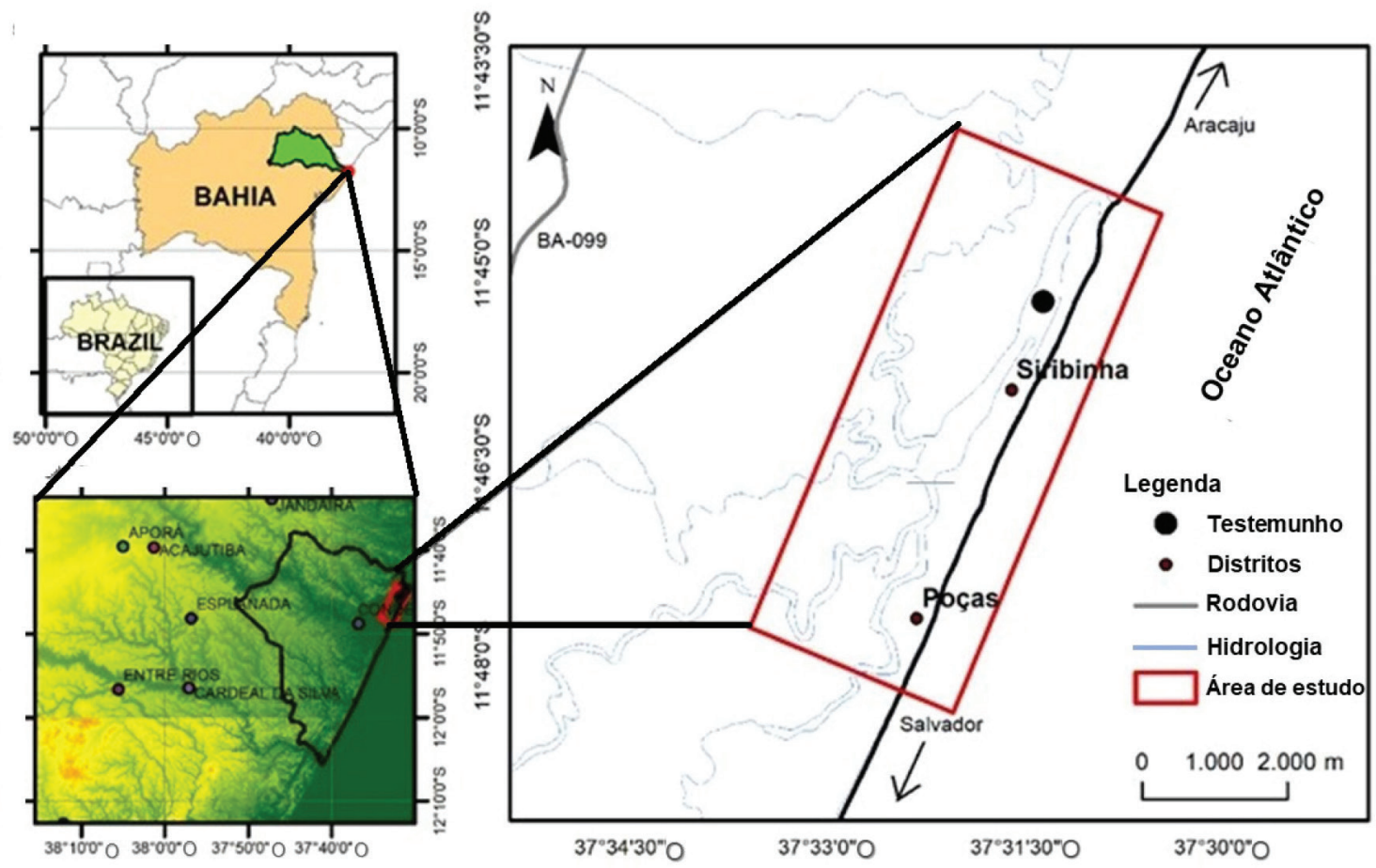

Figura 1. Localização geográfica da bacia hidrogáfica do rio Itapicuru (norte da Bahia), do estuário do rio Itapicuru (Litoral Norte do Estado da Bahia) e do testemunho coletado no presente estudo 
98\% pureza mínima, Synth) foram calcinados a $400{ }^{\circ} \mathrm{C}$ por $4 \mathrm{~h}$ em mufla para eliminação de possíveis interferentes orgânicos. Antes da análise, a sílica e o sulfato de sódio foram ativados em estufa a $150{ }^{\circ} \mathrm{C}$ e resfriados em dessecador, nos quais foram mantidos até serem usados. O hexametafosfato usado nas análises granulométrica foi obtido pela Synth. O cobre usado na eliminação de intereferentes causados pelo enxofre foi tratado com ácido clorídrico $\left(\mathrm{HCl} 2 \mathrm{~mol} \mathrm{~L}{ }^{-1}\right.$, preparado a partir de de uma solução P.A. - Merck), enxaguado com água destilada e emerso em acetona e em DCM. O padrão de $n$-alcanos $n-\mathrm{C}_{8}$ a $n$ - $\mathrm{C}_{40}$ usado na calibração do equipamento e nas fortificações foi da Sigma-Aldrich. Os padrões de Eicoseno e Hexadeceno usados foram obtidos pela SUPELCO (99,7 a 99,9\% de pureza).

Todo material utilizado em laboratório foi deixado em uma solução contendo detergente alcalino Extran (Merck, 5\%) por 12 horas, sendo posteriormente enxaguado com água destilada e seco em temperatura ambiente. Após seco, aquele material destinado para análise elementar e isotópica foi descontaminado com $\mathrm{HCl}\left(10 \%, \mathrm{v} \mathrm{v}^{-1}\right.$, preparado a partir de de uma solução P.A. - Merck) e enxaguado com água Milli-Q. O material não-volumétrico destinado à determinação dos marcadores orgânicos moleculares foi descontaminadas através de calcinação $\left(400^{\circ} \mathrm{C}, 4 \mathrm{~h}\right)$, e o volumétrico, através do enxague com solventes orgânicos (DCM e HEX).

\section{Análise elementar e isotópica}

Para a determinação do carbono orgânico total (COT), nitrogênio total (NT), $\delta^{13} \mathrm{C}$ e $\delta^{15} \mathrm{~N}$, uma pequena quantidade da amostra de sedimento foi homogeneizada e macerada em almofariz de ágata. Cerca de $0,5 \mathrm{~g}$ desta alíquota foi pesada e transferida para tubos Falcon, sendo homogeneizada com $10 \mathrm{~mL}$ de ácido clorídrico $\left(\mathrm{HCl}, 1 \mathrm{~mol} \mathrm{~L}^{-1}\right)$, permanecendo em repouso por mais de 10 horas. As amostras foram centrifugadas à $3000 \mathrm{rpm}$ por cinco minutos, para a retirada do sobrenadante. Esse processo de acidificação das amostras foi repetido três vezes para total eliminação do $\mathrm{CaCO}_{3}$. Posteriormente, foi adicionado $10 \mathrm{~mL}$ de água destilada às amostras, que foram novamente centrifugadas por cinco minutos à $3000 \mathrm{rpm}$. Esse procedimento de lavagem foi repetido três vezes para total eliminação do $\mathrm{HCl}$ (verificada através de fitas de $\mathrm{pH}$ ). As amostras foram secas em estufa a temperaturas entre $45^{\circ}-50{ }^{\circ} \mathrm{C}$.

Entre 10 e $15 \mathrm{mg}$ de cada amostra de sedimento descarbonatado foram pesados e acondicionados em cartuchos de estanho (5 por $8 \mathrm{~mm}$ ) para análise de COT e $\delta^{13} \mathrm{C}$. Para análise de NT e $\delta^{15} \mathrm{~N}$, pesou-se entre 10 e $15 \mathrm{mg}$ de cada amostra sem a descarbonatação. As alíquotas foram submetidas à análise elementar e isotópica em um analisador elementar Costech Instruments Elemental System acoplado a um detector de espectrometria de massa de razão isotópica Thermo Finnigan Delta Plus (EA-IRMS). Os materiais de referência para as análises isotópicas do COT e do NT foram dois padrões certificados pela United States Geological Survey (USGS): USGS-40 (ácido L-glutamínico: $\delta^{13} \mathrm{C}=-26,39 \%$ vs Pee Dee Belemnite - PDB; $\delta^{15} \mathrm{~N}=-4,52 \%$ ov ar) e USGS-41 (ácido L-glutamínico enriquecido em ${ }^{13} \mathrm{C}$ e ${ }^{15} \mathrm{~N}: \delta^{13} \mathrm{C}=+37,63 \%$ vs Pee Dee Belemnite - PDB; $\delta^{15} \mathrm{~N}=+47,57 \%$ ovs ar).

\section{Determinação dos marcadores orgânicos moleculares}

A extração ocorreu em $10 \mathrm{~g}$ de cada amostra, adicionado os padrões internos (1-eicoseno, 1-hexadeceno; $10 \mu \mathrm{L} ; 100 \mu \mathrm{g} \mathrm{mL} \mathrm{m}^{-1}$ ). Adicionou-se às amostras $25 \mathrm{~mL}$ de uma mistura de Hexano: Diclorometano (HEX:DCM; 1:1, v/v), levando-as a um ultrassom, onde permaneceram por 15 minutos $\left(50^{\circ} \mathrm{C}, 35 \mathrm{~Hz}\right)$. Esse procedimento de extração, a partir da adição da mistura HEX:DCM, foi repetido três vezes. As amostras foram filtradas em coluna de vidro (preenchida com sulfato de sódio e algodão limpo) e adicionados fios de cobre ativados para remoção do enxofre elementar, sendo posteriormente pré-concentradas em um evaporador rotativo à vácuo. As soluções resultantes foram submetidas a um processo de purificação em uma coluna de vidro contendo $3 \mathrm{~g}$ de sílica $100 \%$ ativada. Foi utilizado $10 \mathrm{~mL}$ de HEX como fase móvel. A solução final foi pré-concentrada no evaporador rotativo à vácuo e transferidas para microvials. As amostras foram concentradas a $100 \mu \mathrm{L}$ com fluxo de $\mathrm{N}_{2}$. Os marcadores presentes nos extratos orgânicos finais foram identificados e quantificados através de um cromatógrafo a gás (Agilent Technologies modelo 7890B) acoplado a um detector de ionização de chamas (GC-FID). Utilizou-se uma coluna capilar de sílica (HP-1, 15 m x $250 \mu \mathrm{m}$ x 0,25 $\mu \mathrm{m}$ de espessura do filme) para separação dos compostos. A temperatura do sistema de injeção foi programada para $300{ }^{\circ} \mathrm{C}$. A temperatura inicial do forno foi de $40{ }^{\circ} \mathrm{C}$, com posterior aquecimento de $10{ }^{\circ} \mathrm{C} \mathrm{min}-1$ até $300{ }^{\circ} \mathrm{C}$, permanecendo por 12 minutos. Utilizou-se hidrogênio de alto grau de pureza $(99,999 \%)$ como gás de arraste a uma taxa de fluxo de

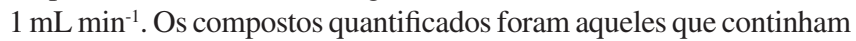
de 15 a 35 átomos de carbono $\left(n-\mathrm{C}_{15}\right.$ a $\left.n-\mathrm{C}_{35}\right)$. A calibração interna do equipamento foi obtida através da injeção de padrão de $n$-alcanos $\left(n-\mathrm{C}_{8}\right.$ a $\left.n-\mathrm{C}_{40}\right)$ com concentrações de 5, 10, 20, 50 e $100 \mathrm{mg} \mathrm{L}^{-1}$. O coeficiente de correlação linear de Pearson desta curva de calibração foi superior a 99,5\% $\left(\mathrm{r}^{2}=0,995\right)$ para todos os compostos analisados. A concentração final do analito foi calculada com base no volume final de extrato e na massa de sedimento seco. O resultado final foi reportado em ng $\mathrm{g}^{-1}$ de sedimento seco (p.s.). A recuperação dos padrões internos esteve entre entre 50 e $150 \%$ em todas as amostras (Material Suplementar, Tabela 1S).

A determinação do limite de detecção do método (LDM) foi baseada em Shrivastava e Grupta. ${ }^{43}$ Ela foi calculada a partir da análise de sete replicatas de sulfato de sódio fortificadas com uma solução padrão $\left(20 \mu \mathrm{L} ; 50 \mu \mathrm{g} \mathrm{mL}^{-1}\right)$ contendo $n$-alcanos $\left(n-\mathrm{C}_{8}\right.$ a $\left.n-\mathrm{C}_{40}\right)$. O LDM foi calculado como três vezes $(3 \mathrm{x})$ o desvio padrão da média dessas análises (Material suplementar, Tabela 2S).

A cada 10 amostras, foi realizada a extração de uma amostra branco $\left(\mathrm{Na}_{2} \mathrm{SO}_{4}\right.$ calcinado) para avaliar a eficiência do método quanto a possíveis fontes de contaminação. Nessas, todos os compostos analisados estiveram menor que LDM. Para o cálculo das razões diagnósticas, aqueles compostos que tiveram suas concentrações abaixo do limite de detecção do método (LDM), o valor encontrado foi substituído pelo valor do LDM dividido por dois.

A análise do padrão de qualidade analítico adotado no presente trabalho foi realizada conforme proposto por CITAC/EURACHEM ${ }^{44} \mathrm{e}$ por Qian e Jackson. ${ }^{45}$ Para avaliar a exatidão do método, quantificou-se uma matriz fortificada. Para isso, adicionaram-se $20 \mu \mathrm{L}$ de um padrão de $n$-alcanos a uma concentração de $50 \mu \mathrm{g} \mathrm{mL}^{-1} \mathrm{em}$ uma amostra sedimentar. Essa amostra foi submetida a todo método acima descrito. Mais de $80 \%$ dos compostos apresentaram recuperação entre 50 e $120 \%$, com exceção do $n-\mathrm{C}_{27}, n-\mathrm{C}_{29}$ e $n$ - $\mathrm{C}_{31}$ (Material Suplementar, Tabela 1S). A concentração desses compostos na amostra foi mais que quatro vezes superior ao que foi adicionado na fortificação, o que deve ter ocasionado essa diferença. Foi realizada a análise de duas amostras em duplicata, na qual $80 \%$ dos compostos apresentaram desvio padrão relativo acima de 30\% (Material Suplementar, Tabela 1S).

\section{Geocronologia}

A atividade de ${ }^{210} \mathrm{~Pb}$ foi determinada conforme método proposto por Godoy et al. ${ }^{46} \mathrm{O}$ detector proporcional de fluxo gasoso utilizado foi da marca Canberra modelo S5 XLB. O modelo de concentração inicial constante (modelo-CIC) foi utilizado para a obtenção da taxa de sedimentação do testemunho, por meio do decaimento radioativo 
de ${ }^{210} \mathrm{~Pb}^{47,48}$ Esse modelo foi escolhido porque supõe-se que a área estudada é estável, sem muitas mudanças na taxa de sedimentação e a incorporação do ${ }^{210} \mathrm{~Pb}$ em excesso ou não suportado aos sedimentos ocorre a um fluxo constante. Para se estimar a datação do testemunho utiliza-se da seguinte equação: data estimada $=a-(b / c)$, em que 'a' é o ano que o testemunho foi coletado, 'b' é a profundidade da seção a ser analisada e 'c' é a taxa de sedimentação encontrada no testemunho.

\section{Análise granulométrica}

Pesou-se aproximadamente $1,0 \mathrm{~g}$ de cada sub-amostra, sendo a alíquota peneirada $(\varphi=0,425 \mathrm{~mm})$ para separação da areia grossa $(2-0,5 \mathrm{~mm})$. O procedimento dessa análise granulométrica foi realizada segundo Garcia et al., ${ }^{49}$ e a determinação do tamanho das partículas das amostras de sedimentos foi realizada em analisador de partículas com difração a laser (modelo Cilas 1064) na faixa granulométrica de $0,004-0,5 \mathrm{~mm}$, correspondendo as frações areia média $(0,5-0,25 \mathrm{~mm})$, areia fina $(0,25-0,13 \mathrm{~mm})$, areia muito fina $(0,13-0,063 \mathrm{~mm})$, silte $(0,063-0,004 \mathrm{~mm})$ e argila $(>0,004 \mathrm{~mm}){ }^{50}$

\section{Análise estatística}

O software BioEstat 5.3 foi usado para as análises estatísticas descritivas, sendo que a correlação de Spearman foi utilizada devido aos dados serem considerados não-normais. Essas correlações foram consideradas significativas quando $\mathrm{p}<0,05$.

\section{RESULTADOS E DISCUSSÃO}

\section{Taxa de sedimentação e análise granulométrica}

A taxa de sedimentação do testemunho analisado foi de $0,54 \pm 0,05 \mathrm{~cm}^{2} \mathrm{ano}^{-1}$. Esse valor coincide com o encontrado por Farias, ${ }^{37}$ que também verificou que na região de estudo a taxa de sedimentação era de $0,54 \mathrm{~cm} a^{-1}$. A base do testemunho teve sua data estimada em 1907 (base). Assim, o período de abragência estimada para o testemunho coletado é de aproximadamente em 110 anos. O erro associado à geocronologia foi estimado em 0,1 ano para cada camada de $2 \mathrm{~cm}$ de sedimentos depositados.

A fração lamosa foi minoritária ao longo de todo o testemunho, com valores variando de 6,44 a $22,7 \%$ (Tabela 1 ). Isso ocorre devido à características do estuário em questão e a localização do ponto de coleta. ${ }^{31,37,51}$ A região mais externa do estuário está próxima a um sistema de dunas, onde há predomínio de areia. Além disso, a região de coleta é dominada por ondas e marés, que aumentam a hidrodinâmica local $^{37,52,53} \mathrm{e}$, por consequência, a proporção de areia. ${ }^{54}$

\section{Análise quantitativa da matéria orgânica depositada}

Na Tabela 1, estão apresentados os valores de COT (\%), NT (\%) e AlcTot (somatório das concentrações dos $n$-alcanos totais) encontrados para as sub-amostras avaliadas no testemunho coletado. Os teores variaram de 0,65 a 2,51\%, 0,07 a $0,13 \%$ e 1.331 a $7.384 \mathrm{ng} \mathrm{g}^{-1}$ em peso seco (p.s.), respectivamente. Observou-se uma correlação positiva significativa $(\mathrm{p}<0,05)$ entre a quantidade de lama e as concentrações de COT $(r=0,81)$, NT $(r=0,70)$ e AlcTot $(r=0,88)$. Essa tendência pode ser atribuída à relação de maior adsorção de matéria orgânica ao sedimento mais fino. ${ }^{55}$ Assim, a variação nas concentrações do COT, NT e AlcTot tendem a seguir as mudanças na porcentagem de sedimento fino ao longo do testemunho. Na Figura 2, tem-se os perfis encontrados para essas quatro variáveis. Observa-se que há um aumento do teor de lama no testemunho como um todo, porém, com algumas oscilações. Devido a sua correlação positiva com o COT, NT e AlcTot, esse aumento interfere diretamente nas concentrações dos demais parâmetros. Da base (1907) a 44 cm (1936), o sedimento apresentou os menores teores de lama e as concentrações de AlcTot tenderam a uma leve diminuição (de 2030 a 1331 ng g ${ }^{-1}$ p.s.). Já os teores de COT e NT seguiram a mesma tendência do sedimento fino. A partir de $44 \mathrm{~cm}$ (1936) até $24 \mathrm{~cm}$ (1973), houve um pequeno aumento das concentrações de COT, NT e AlcTot. Até o início dos

Tabela 1. Profundidade da seção no testemunho coletado (cm), data estimada (anos), conteúdo de silte + argila, (Lama, \%), teor do carbono orgânico total (COT, $\%$ ), teor do nitrogênio total (NT, \%), somatório dos $n$-alcanos totais (AlcTot, ng g $\mathrm{g}^{-1}$ ), razões isotópicas do carbono orgânico total ( $\delta^{13} \mathrm{C}$, \%o) e do nitrogênio total $\left(\delta^{15} \mathrm{~N}, \%\right.$ ) , e seus respectivos valores mínimo (Mín.), máximo (Máx.), médio (Méd.) e desvio padrão (DP) encontrados nas sub-amostras avaliadas no testemunho coletado às margens do estuário do rio Itapicuru (BA)

\begin{tabular}{|c|c|c|c|c|c|c|c|}
\hline Seção (cm) & $\begin{array}{l}\text { Data estimada } \\
\quad(\text { anos })\end{array}$ & Lama (\%) & $\operatorname{COT}(\%)$ & NT $(\%)$ & AlcTot (ng g-1) & $\delta^{13} \mathrm{C}(\%)$ & $\delta^{15} \mathbf{N}(\% o)$ \\
\hline 0 & 2017 & 22,0 & 2,39 & 0,11 & 7384 & $-28,1$ & 1,87 \\
\hline 4 & 2010 & 19,7 & 2,32 & 0,13 & 5363 & $-26,8$ & 0,35 \\
\hline 8 & 2002 & 18,4 & 1,92 & 0,11 & 4012 & $-26,6$ & $-0,54$ \\
\hline 12 & 1995 & 17,0 & 1,66 & 0,11 & 4091 & $-26,9$ & $-1,36$ \\
\hline 16 & 1988 & 18,6 & 2,14 & 0,12 & 3808 & $-25,8$ & 0,97 \\
\hline 24 & 1973 & 22,7 & 2,51 & 0,13 & 4136 & $-25,2$ & 0,75 \\
\hline 28 & 1965 & 16,2 & 2,35 & 0,12 & 3594 & $-24,8$ & $-0,30$ \\
\hline 32 & 1958 & 16,0 & 2,10 & 0,11 & 3329 & $-24,3$ & $-0,87$ \\
\hline 36 & 1951 & 14,8 & 2,13 & 0,11 & 2751 & $-24,9$ & 0,06 \\
\hline 40 & 1943 & n.a. & 1,70 & 0,09 & 2127 & $-25,1$ & 0,27 \\
\hline 44 & 1936 & 16,0 & 2,05 & 0,11 & 1331 & $-26,6$ & $-1,11$ \\
\hline 48 & 1929 & 10,8 & 1,40 & 0,09 & 1516 & $-27,8$ & $-0,73$ \\
\hline 52 & 1921 & 6,44 & 0,65 & 0,07 & 1582 & $-28,2$ & $-6,23$ \\
\hline 56 & 1914 & 12,4 & 1,09 & 0,08 & 1922 & $-28,8$ & $-1,55$ \\
\hline 60 & 1907 & 13,9 & 1,18 & 0,08 & 2030 & $-28,5$ & 0,32 \\
\hline Mín. & - & 6,44 & 0,65 & 0,07 & 1331 & $-28,8$ & $-6,23$ \\
\hline Máx. & - & 22,7 & 2,51 & 0,13 & 7384 & $-24,3$ & $\mathbf{1 , 8 7}$ \\
\hline Méd. & - & 16,1 & 1,84 & $\mathbf{0 , 1 0}$ & 3486 & $-26,6$ & $-0,54$ \\
\hline DP & - & 4,34 & 0,55 & 0,02 & 1825 & 1,48 & $\mathbf{1 , 8 3}$ \\
\hline
\end{tabular}


anos 70, não havia um considerado desenvolvimento urbano no entorno da bacia hidrográfica do rio Itapicuru, tampouco na região do seu estuário. Pode-se considerar que as variações na parte mais profunda do testemunho são ocasionadas por fenômenos naturais, como mudanças em correntes, padrões de vento e precipitação.

Após esse período, pode-se verificar uma redução das concentrações de todas as variáveis até entorno de 1995, seguido de um aumento das concentrações do teor de lama, de COT e AlcTot no testemunho. No início dos anos 70, já se iniciava na região do Litoral Norte baiano o incentivo ao reflorestamento, com o estímulo do plantio de pinus e eucalipto para a indústria de celulose e ao processo de ocupação. ${ }^{29}$ Com essas práticas, há uma declínio da cobertura vegetal e denudação de solos arenosos, disponibilizando-os e, por consequência, depositando-os nos sistemas aquáticos adjacentes. Essa prática foi uma das grandes responsáveis pela perda da biodiversidade local ${ }^{29}$ Além disso, na década de 70 houve o processo de ocupação e retirada das áreas de restingas, através da implantação de loteamentos na região. ${ }^{29}$ Isso pode ter ocasionado uma diminuição no aporte sedimentar e da quantidade de M.O. para região. De 1995 até 2013, houve um novo aumento na quatidade de matéria orgânica depositada. A partir de 1993, houve a ampliação da rodovia BA-099 (Linha Verde), que corta o Litoral Norte baiano até a divisa com o estado de Sergipe. Essa foi uma das principais intervenções antrópicas feita na região. Com a construção dessa estrada, houve um aumento erosivo dos sedimentos juntamente com a retirada da cobertura vegetal, aumentando assim o aporte de M.O. para região estudada. ${ }^{56}$

\section{Análise qualitativa da matéria orgânica depositada}

Os valores das razões isotópicas de carbono e nitrogênio variaram de $-28,8$ a $-24,3 \%$ o e de $-6,23$ a 1,83\%o, respectivamente. Estes valores são típicos de plantas terrígenas com padrão fotossintético do tipo $\mathrm{C}_{3}\left(\delta^{13} \mathrm{C}\right.$ entre $-33 \%$ e e $-22 \%$ e $\delta^{15} \mathrm{~N}$ próximo a $0 \%$ o $){ }^{9,10}$ A região de estudo está envolta por uma Mata Atlântica bem desenvolvida com a presença de vegetação de mata ciliar e de mangue. A Rhizophora mangle e a Laguncularia racemosa são as espécies dominantes no mangue adjacente ao estuário em questão. Já as espécies da família Polygonnaceae e Myrtaceae dominam a vegetação de Mata Atlântica da região. ${ }^{42}$ Todas elas apresentam padrão fotossintético do tipo $\mathrm{C}_{3}$.

Os dados de $n$-alcanos analisados, confirmam que a composição orgânica da região estudada é dominada por plantas superiores. Em todo o testemunho foi observado uma maior concentração de compostos de cadeias carbônicas longas $\left(n-\mathrm{C}_{25}-n-\mathrm{C}_{35}\right)$ que curtas $\left(n-\mathrm{C}_{15}-n-\mathrm{C}_{23}\right.$ ), principalmente o $n-\mathrm{C}_{27}, n-\mathrm{C}_{29}$ e $n$ - $\mathrm{C}_{31}$ (Tabela 1S). As maiores concentrações se dão ao $n-\mathrm{C}_{29}$, seguido de $n-\mathrm{C}_{27}$ e/ou $n-\mathrm{C}_{31}$, comum em vegetações do como o padrão fotossintético do tipo $\mathrm{C}_{3} \cdot{ }^{20,21}$ Baseado nas distribuições dos compostos encontrados, calculou-se razões diagnósticas para verificar as diferentes fontes de M.O. para a área de estudo (Tabela 2).

Os valores da razão da somatória dos compostos de baixa massa molecular sobre os de alta massa molecular (BMM/AMM) e os do Índice Preferencial de Carbono (CPI) variaram de 0,17 a 0,93 e de de 2,79 a 13,1, respectivamente, sugerindo que a M.O. depositada no estuário do rio Itapicuru tem como principal origem as plantas superiores. ${ }^{57,59}$ Utilizando os dados de CPI, pode-se classificar a M.O. presente como provenientes de monocotiledônea $(<5)$ e dicotiledônea ( $>5) .{ }^{22}$ Ao se observar a Tabela 2, os valores de CPI de grande parte das amostras se mantiveram abaixo de 5, o que mostra uma maior contribuição de plantas superiores provindas do grupo de monocotiledôneas, como as espécies Eichornia crassipes e Spartina alterniflora. $^{32}$

O tamanho médio das cadeias (ACL) descreve o número médio de átomos de carbono presente nas moléculas de $n$-alcanos detectados em um ambiente. ${ }^{4,58,60} \mathrm{O}$ Índice Alcano (AI) é baseado nas diferenças dos $n$-alcanos de origem terrígena $\left(n-\mathrm{C}_{31}\right.$ e $n$ - $\left.\mathrm{C}_{29}\right)$. Plantas vasculares do tipo $\mathrm{C}_{3}$ tendem a produzir $n$-alcanos de cadeias que estão entre de $n$ - $\mathrm{C}_{27}$ e $n$ - $\mathrm{C}_{29}$. Já as plantas do tipo $\mathrm{C}_{4}$ produzem maiores quantidades relativas de $n-\mathrm{C}_{31}$ e $n-\mathrm{C}_{33}{ }^{21}{ }^{21}$ Segundo Albergaria-Barbosa, ${ }^{22}$ utilizando os valores de ACL e AI, é possível classificar as plantas superiores em vegetação de mangue ou ribeirinha. Os valores de ACL $<29,0$ são indicativos de fonte de vegetação de mangue. Já valores $>29,0$ são para vegetação ribeirinha. Nesse estudo, os valores de ACL e de AI variaram de 26,9 a 28,9 e de 0,21 a 0,63 , respectivamente, mostrando uma maior contribuição de vegetação ribeirinha. ${ }^{22} \mathrm{~A}$ região de estudo está envolta por uma Mata Atlântica bem desenvolvida com a presença de vegetação de mata ciliar e de mangue. Essa é dividida em ombrófila densa, mata ciliar, mangue e restinga. A região possui o maior manguezal da APA/LN, com uma área de aproximadamente 900 km² de vegetação lenhosa da Mata Atlântica (manguezal) em torno do estuário. ${ }^{32,39} \mathrm{~A}$ mata ciliar do município de Conde não foi muito estudada. Essa, entretanto, mostra um elevado estágio de regeneração, sugerindo a presença de endemismos. ${ }^{29}$

Os valores do índice de produção aquática (Paq) expressa a proporção entre produtores sintetizantes de cadeia média $\left(n-\mathrm{C}_{23} \mathrm{e}\right.$ $\left.n-\mathrm{C}_{25}\right)$ e os produtores sintetizantes de cadeia longa $\left(n-\mathrm{C}_{29}\right.$ e $\left.n-\mathrm{C}_{31}\right)$, que correspondem às macrófitas aquáticas e às plantas superiores, respectivamente. ${ }^{20,24}$ Para este parâmetro também pode ser feita a classificação provinda de monocotiledônea e dicotiledônea. ${ }^{22}$ Os

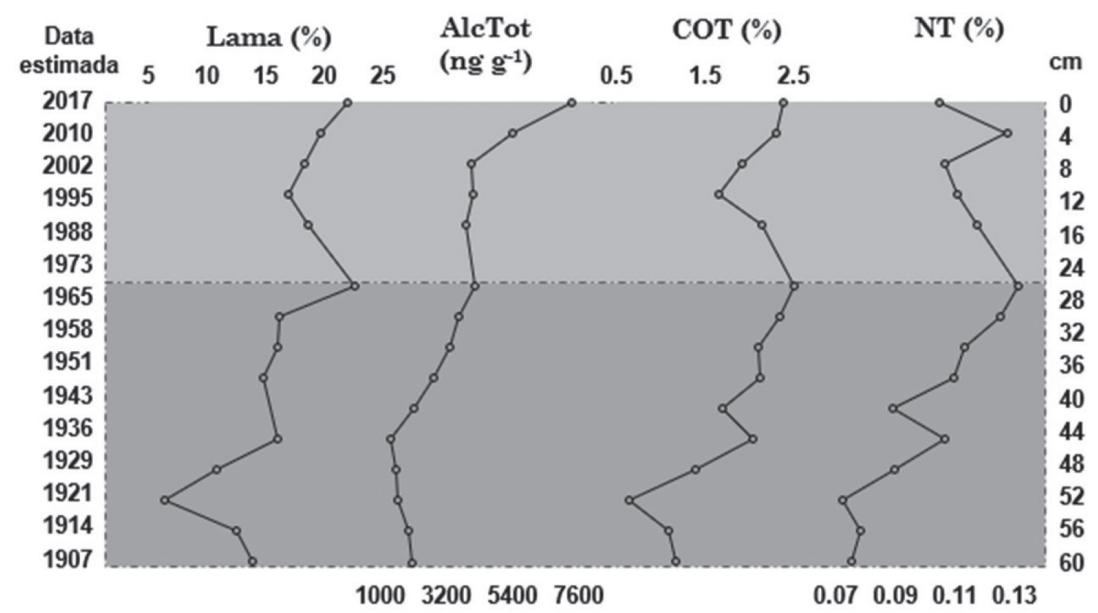

Figura 2. Perfil vertical dos teores de lama (\%), da concentração de n-alcanos totais (AlcTot, ng g $g^{-1}$ em peso seco), dos teores de carbono orgânico total (COT, $\%)$ e de nitrogênio total (NT, \%) do testemunho coletado no estuário do rio Itapicuru (BA) 
Tabela 2. Data estimada, seção do testemunho (cm), somatório dos $n$-alcanos de baixa massa molecular (BMM $\leq n$ - $C_{25}$, ng g ${ }^{-1}$ ), somatório dos $n$-alcanos de alta massa molecular (AMM $\left.\leq n-\mathrm{C}_{25}, \mathrm{ng} \mathrm{g}^{-1}\right), n$-alcanos de baixa massa molecular sobre os de alta massa molecular (BPM/APM), CPI (Índice Preferencial do Carbono), Paq (Índice de Produção Aquática), ACL (Tamanho Médio da Cadeia) e AI (Índice Alcano), e seus respectivos mínimo (mín.), máximo (máx.) e desvio padrão (DP) do testemunho coletado às margens do estuário do rio Itapicuru (BA)

\begin{tabular}{|c|c|c|c|c|c|c|c|c|}
\hline Data estimada & Seção $(\mathrm{cm})$ & $\mathbf{B M M} \leq \boldsymbol{n}-\mathrm{C}_{25}$ & $\mathrm{AMM} \geq \boldsymbol{n}-\mathrm{C}_{25}$ & BMM/AMM & CPI & Paq & ACL & AI \\
\hline 2017 & 0 & 1812 & 5977 & 0,30 & 2,84 & 0,16 & 28,7 & 0,31 \\
\hline 2010 & 4 & 1267 & 4423 & 0,29 & 2,79 & 0,17 & 28,7 & 0,34 \\
\hline 2002 & 8 & 915 & 3342 & 0,27 & 2,98 & 0,16 & 28,7 & 0,34 \\
\hline 1995 & 12 & 916 & 3414 & 0,27 & 3,05 & 0,15 & 28,7 & 0,31 \\
\hline 1988 & 16 & 699 & 3337 & 0,21 & 3,12 & 0,15 & 28,8 & 0,38 \\
\hline 1973 & 24 & 872 & 3534 & 0,25 & 2,97 & 0,17 & 28,7 & 0,38 \\
\hline 1965 & 28 & 561 & 3253 & 0,17 & 2,94 & 0,15 & 28,9 & 0,39 \\
\hline 1958 & 32 & 699 & 2819 & 0,25 & 2,94 & 0,15 & 28,7 & 0,34 \\
\hline 1951 & 36 & 802 & 2090 & 0,38 & 3,47 & 0,15 & 28,7 & 0,32 \\
\hline 1943 & 40 & 639 & 1609 & 0,40 & 4,91 & 0,15 & 28,5 & 0,21 \\
\hline 1936 & 44 & 252 & 1147 & 0,22 & 6,02 & 0,12 & 28,7 & 0,22 \\
\hline 1929 & 48 & 363 & 1270 & 0,29 & 3,91 & 0,19 & 28,4 & 0,22 \\
\hline 1921 & 52 & 683 & 1044 & 0,65 & 4,54 & 0,28 & 28,1 & 0,63 \\
\hline 1914 & 56 & 960 & 1400 & 0,69 & 6,99 & 0,55 & 26,8 & 0,21 \\
\hline 1907 & 60 & 1150 & 1236 & 0,93 & 13,1 & 0,49 & 26,8 & 0,21 \\
\hline Mín. & & 252,3 & 1044 & 0,17 & 2,79 & 0,12 & 26,8 & 0,21 \\
\hline Máx. & & 1812 & 5977 & 0,93 & 13,1 & 0,55 & 28,9 & 0,63 \\
\hline Méd. & & 839,2 & 2660 & 0,37 & 4,44 & 0,21 & 28,4 & 0,32 \\
\hline DP & & 378,6 & 1430 & 0,22 & 2,72 & 0,13 & 0,68 & 0,11 \\
\hline
\end{tabular}

$B M M / A M M=\sum n C_{15-25} / \sum n C_{25-35}{ }^{57} ; P a q=\left[\frac{n \mathrm{C}_{23}+n \mathrm{C}_{25}}{n \mathrm{C}_{23}+n \mathrm{C}_{25}+n \mathrm{C}_{29}+n \mathrm{C}_{31}}\right]^{20} ; A C L=\frac{\sum\left[n \mathrm{C}_{i} \times i\right]^{58}}{\sum\left[n \mathrm{C}_{i}\right]} ; A I=\frac{\left[n \mathrm{C}_{31}\right]}{\left[n \mathrm{C}_{29}+n \mathrm{C}_{31}\right]}{ }^{21}$;

$C P I=0,5 x\left[\frac{n \mathrm{C}_{25}+n \mathrm{C}_{27}+n \mathrm{C}_{29}+n \mathrm{C}_{31}+n \mathrm{C}_{33}}{n \mathrm{C}_{24}+n \mathrm{C}_{26}+n \mathrm{C}_{28}+n \mathrm{C}_{30}+n \mathrm{C}_{32}}+\frac{n \mathrm{C}_{25}+n \mathrm{C}_{27}+n \mathrm{C}_{29}+n \mathrm{C}_{31}+n \mathrm{C}_{33}}{n \mathrm{C}_{26}+n \mathrm{C}_{28}+n \mathrm{C}_{30}+n \mathrm{C}_{32}+n \mathrm{C}_{34}}\right]^{59}$

valores de Paq para a região estudada variaram entre 0,12 a 0,55 , mostrando maior contribuição de plantas monocotiledôneas $(>0,1)$ na matéria orgânica depositada, principalmente na base do testemunho. ${ }^{22}$

Santana ${ }^{51}$ também observou a influência de monocotiledôneas/ macrófitas em algumas áreas do estuários do rio Itapicuru. Segundo o $\mathrm{CRA}^{32}$ espécies como orelha-de-burro (Pontederia lanceolata), aguapé (Eichhornia crassipes), briófitas-veludo (Sphagnum sp) e (Spartina alterniflora) são comuns na região.

Os perfis verticais dos valores das razões analisadas no testemunho coletado são mostrados na Figura 3. Para a razão $\delta^{13} \mathrm{C}$, existe uma variação ao longo de sua distribuição vertical, embora os valores encontrados sejam típicos de planta terrígena. O $\delta^{15} \mathrm{~N}$ segue a mesma tendência dos teores de lama e COT. Existe uma correlação positiva significativa do $\delta^{15} \mathrm{~N}$ com essas outras variáveis $(\mathrm{r}=0,70$, $\mathrm{p}<0,004 ; \mathrm{r}=0,71, \mathrm{p}<0,003$; respectivamente). Os perfis das razões dos $n$-alcanos mostram que houve para o estuário do rio Itapicuru dois momentos distintos de deposição. Da base (1907) até 40 cm (1943), os valores de Paq e CPI diminuiram, mostrando a troca no predomínio da matéria orgânica provinda de macrófitas para aquela provinda de vegetação superior. ${ }^{22}$ Os valores de ACL também aumentaram, mostrando um aumento da influência da vegetação superior ribeirinha na matéria orgânica depositada. ${ }^{22}$ Durante esse período, a região de estudo encontrava-se sob baixo desenvolvimento urbano. $\mathrm{O}$ acesso a essa região era baixo e suas vilas/cidades eram pequenas. ${ }^{29}$ Assim, as observações encontradas podem ter sido causadas por fenômenos naturais. As macrófitas geralmente se desenvolvem em regiões litorâneas de ambientes quentes, com alta produção de nutrientes e com baixa hidrodinâmica, principalmente nas partes mais interna do estuário. ${ }^{32}$ Provavelmente, durante este período, houve uma mudança na hidrodinâmica da região que resultou nas alterações das principais fontes de matéria orgânica para a região.

A partir de $40 \mathrm{~cm}$ (1943), as razões BMM/AMM, CPI, Paq e ACL se mantiveram relativamente constantes, mostrando assim que não houve uma mudança no tipo de matéria orgânica que foi depositada na região estuarina do rio Itapicuru desde esse período até 2017. Sabe-se que no entorno do estuário do rio Itapicuru, a vegetação de Mata Atlântica tem sido substituída por plantas não nativas. O município de Conde tem como principal base econômica as atividades agropecuárias. A partir da década de 70, com o incentivo por parte do governo estadual para a indústria de celulose, houve nos entornos do município de Conde o plantio de pinus e eucaliptos. A taxa de reflorestamento do município para esta atividade é a segunda maior do litoral norte (cerca de 10\%). ${ }^{29}$ Além de pinus, ocorre também na região o plantio de coco. Cerca de $70 \%$ do coqueiral nordestino estão concentrados nos municípios litorâneos da Bahia, onde Conde se sobressai como o principal produtor de coco do estado. Há ainda, em menor escala, a cultura de maracujá, manga, laranja e banana (da terra e prata). A produção dessa última tem crescido nas últimas décadas, sendo usada tanto para consumo local quanto para comercialização. ${ }^{29}$ Após a construção da linha verde, houve um rápido crescimento urbano que, com os loteamentos, gerou um processo de degradação acelerado das áreas de restinga. 


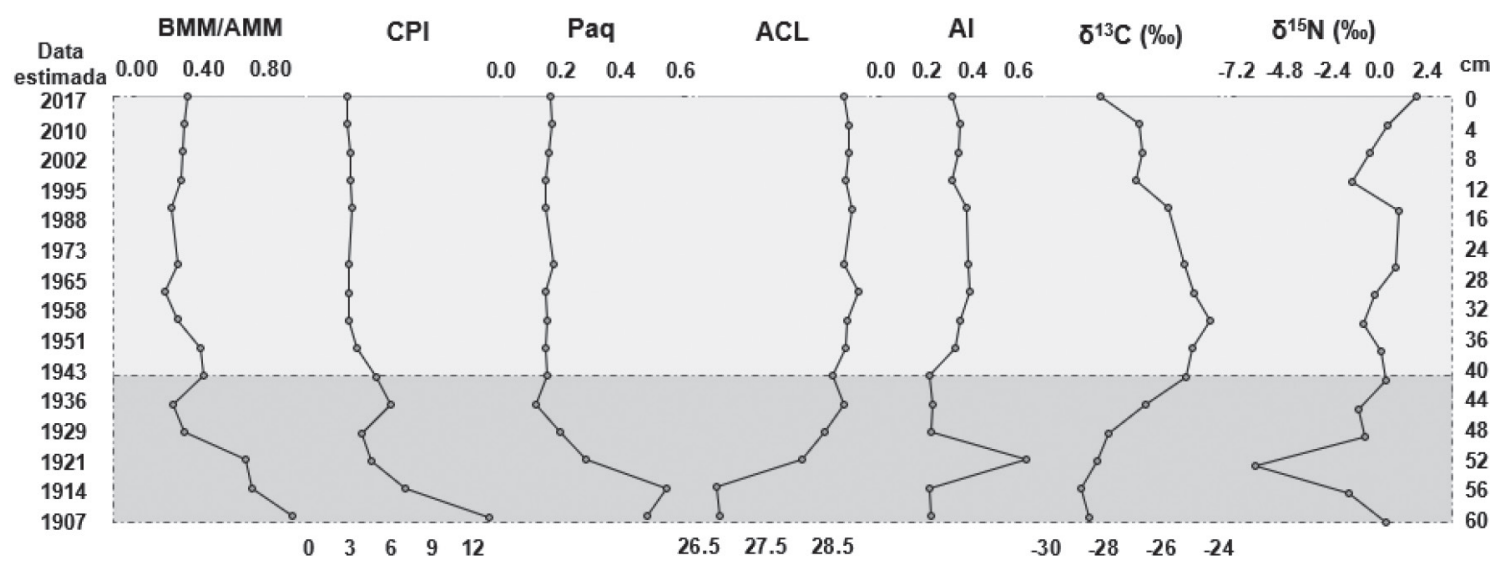

Figura 3. Perfil vertical dos valores das razões de n-alcanos de baixa massa molecular sobre os de alta massa molecular (BMM/AMM), do índice preferencial de carbono (CPI), do índice de produção aquática (Paq), do tamanho médio da cadeia de n-alcanos (ACL), do índice alcano (AI) e da razão isotópica do carbono $\left(\delta^{13} \mathrm{C}\right)$ e do nitrogênio $\left(\delta^{15} \mathrm{~N}\right)$ para o testemunho coletado no estuário do rio Itapicuru $(B A)$

A agropecuária, a extração de areia, a construção de estradas e a deposição de lixo também se apresentam como tensores para esta vegetação. ${ }^{29}$ Apesar de todos esses processos ocasionarem mudanças no tipo de vegetação da região, esse não pode ser observado usando-se os marcadores moleculares estudados no presente estudo.

\section{CONCLUSÕES}

Este estudo identificou mudanças temporais do aporte de matéria orgânica para o estuário do rio Itapicuru. As concentrações de matéria orgânica depositada no sistema em questão estão relacionadas diretamento com a granulometria local. Os marcadores analisados não conseguiram avaliar a influência da troca da vegetação nessas fontes. Aplicando as razões isotópicas e as razões diagnósticas dos $n$-alcanos, pode-se confirmar que as principais fontes de M.O. para a região de estudo no períodos de abrangência do testemunho coletado são as macrófitas e as plantas terrígenas vasculares durante toda a data estimada do testemunho coletado, havendo leves oscilações. Essas principais mudanças observadas nas fontes de matéria orgânica ocorrem antes do desenvolvimento urbano da região. Assim, suas principais causas devem ser naturais.

\section{MATERIAL SUPLEMENTAR}

As Tabelas 1S e 2S estão disponíveis em http://quimicanova.sbq. org.br, em formato PDF, com acesso livre.

\section{AGRADECIMENTOS}

O presente trabalho foi realizado no âmbito do Programa de Pós-Graduação em Geoquímica: Petróleo e Meio Ambiente da Universidade Federal da Bahia (POSPETRO/UFBA) com o apoio da Coordenação de Aperfeiçoamento de Pessoal de Nível Superior - Brasil (CAPES) - Código de Financiamento 001. Agradecemos à Pró-Reitoria de Pesquisa, Criação e Inovação da Universidade Federal da Bahia (PROPCI-PROPG/UFBA-04/2014 - PRODOC/ UFBA edital No 014/2014) e à Fundação de Amparo a Pesquisa do Estado da Bahia (FAPESB, CNV0005/2013; CNV0025/2013) pelo financiamento dos equipamentos utilizados.

\section{REFERÊNCIAS}

1. Tesi T.; Miserocchi, S.; Goñi, M. A.; Langone, L.; Boldrin, A.; Turchetto, M.; Estuarine, Coastal Shelf Sci. 2007, 73, 431.
2. Dietrich, L. A. S.; Mcinnis, D. P.; Bolster, D.; Maurice, P. A.; Water Res. 2013, 47, 2231.

3. Mcinnis, D. P.; Bolster, D.; Maurice, P. A.; Environ. Eng. Sci. 2014, 31, 98.

4. Derrien, M.; Yang, L.; Hur, J.; Water Res. 2017, 112, 58.

5. Lehrback, B. D.; Neto, R. R.; Barroso, G. F.; Bernardes, M. C.; Braz. J. Aquat. Sci. Technol. 2016, 20, 79.

6. Remeikaitė-Nikienė, N.; Lujanienė, G.; Malejevas, V.; Barisevičiūtė, R.; Žilius, M.; Garnaga-Budrè, G.; Stankevičius, A.; Journal of Marine Systems 2016, 157, 75.

7. Hu, L.; Guo, Z.; Feng, J.; Yang, Z.; Fang, M.; Mar. Geochem. 2009, 113, 197.

8. Hu, J.; Peng. P.; Jia, G.; Mai, B.; Zhang, G.; Mar. Geochem. 2006, 98, 274.

9. Meyers, P. A.; Org. Geochem. 1997, 27, 213.

10. Pancost, R. D.; Boot, C. S.; Mar. Chem. 2004, 92, 239.

11. Barros, G. V.; Martinelli, L. A.; Novais, T. M. O.; Omettoj. P. H. B.; Zuppi, G. A.; Sci. Total Environ. 2010, 408, 2226.

12. Xia, B.; Cui, Y.; Chen, B.; Cui, Z.; Qu, K.; Ma, F.; Acta Oceanol. Sin. 2014, 33, 48.

13. Meyers, P. A.; Chem. Geol. 1994, 114, 289.

14. Harji, R. R.; Yvenat, A.; Bhosle, N. B.; Environ. Int. 2008, 34, 959.

15. Silva, T. R.; Lopes, S. R. P.; Sporl, G.; Knoppers, B. A.; Azevedo, D. A.; Org. Geochem. 2012, 53, 25.

16. Ranjan, R. K.; Routh, J.; Klump, J. V.; Ramanathan A. L.; Mar. Chem. 2015, 171, 44.

17. Souza, J. R. B.; Zuchi, M. R.; Costa, A. B.; Azevedo, A. E. X.; Spano, S.; Mar. Pollut. Bull. 2017, 119, 239.

18. Volkman, J. K.; Holdsworth, D. G.; Neill, G. P.; Sci. Total Environ. 1992, 112, 203.

19. Eglinton, T. I.; Eglinton, G.; Earth Planet Sci. Lett. 2008, 275, 1.

20. Ficken, K. J.; Li, B.; Swain, D. L.; Eglinton, G.; Org. Geochem. 2000. 31,745 .

21. Rommerskirchen, F.; Eglinton, G.; Dupont, L.; Güntner, U.; Wenzel, C.; Rullkötter, J.; Geochem., Geophys., Geosyst. 2003, 4, 1.

22. Albergaria-Barbosa, A. C. R.; Tese de doutorado, Universidade de São Paulo, Brasil, 2013.

23. Wang, S.; Liu, G.; Yuan, Z.; Da, C.; Ecotoxicol. Environ. Saf. 2018, 150, 199.

24. Sikes, E. L.; Uhle, M. E.; Nodder, S. D.; Howard, M. E.; Mar. Chem. 2009, 113, 149.

25. Killops, S.; Killops, V.; Introduction to Organic Geochemistry, $2^{\text {nd }}$ ed., Oxford: New York, 2005.

26. Inema - Instituto do Meio Ambiente e Recursos Hídricos, disponível 
em http://www.inema.ba.gov.br/gestao-2/unidades-de-conservacao/apa/ apa-litoral-norte-do-estado-da-bahia, acessada Maio de 2019.

27. Ribeiro, C. A. O.; Dissertação de mestrado, Universidade Federal da Bahia, Brasil, 2006.

28. Virães, M. V.; Regionalização de Vazões nas Bacias Hidrográficas Brasileiras: estudo da vazão de $95 \%$ de permanência da sub-bacia 50 - Bacias dos rios Itapicuru, Vaza Barris, Real, Inhambupe, Pojuca, Sergipe, Japaratuba, Subaúma e Jacuípe - Recife: CPRM, 2013, 154p.

29. CRA - Centro de Recursos Ambientais, Projeto de Gerenciamento Costeiro. Diagnóstico oceanográfico e proposição de disciplinamento de usos da faixa marinha do Litoral Norte do estado da Bahia, 2003, Relatório técnico, $128 \mathrm{p}$

30. Muricy, I. T.; Anais do XVII Encontro Nacional de Estudos Populacionais, Caxambu, Brasil, 2010.

31. Silva, A. M. M. A.; Monografia de graduação, Universidade Federal da Bahia, Brasil, 2009.

32. CRA - Centro de Recursos Ambientais; Projeto de gerenciamento costeiro. Gestão integrada da orla marítima no município do Conde no estado da Bahia. Diagnóstico sócio e econômico ambiental do Conde, 2005, Relatório técnico, 221p.

33. Lima, G. M.; Dissertação de mestrado, Universidade Federal da Bahia, Brasil, 2007.

34. Bittencourt, A. C. S. P.; Livramento, F. C.; Dominguez, J. M. L.; Silva, I. R.; Rev. Bras. Geociênc. 2010, 40, 125.

35. Netto, M. A.; Em Litoral norte da Bahia: caracterização ambiental, biodiversidade e conservação; Nunes, J. M. C., Matos, M. R. B., eds., Edufba: Salvador, 2017, cap. 1.

36. Dominguez J. M. L., Leão Z. M. A. N., Lyrio R. S.; $39^{\circ}$ Congresso Brasileiro Geologia, Salvador, Brasil, 1996.

37. Farias, F. F.; Tese de doutorado, Universidade Federal da Bahia, Brasil, 2014.

38. Correia, I. O.; Monografia de graduação, Instituto de Geociências, Universidade Federal da Bahia, Brasil, 2017.

39. Esqueviel, M. S.; Dissertação de mestrado, Universidade Federal da Bahia, Brasil, 2006

40. Alvares, C. A.; Stape, J. L.; Sentelhas, P. C.; Gonçalves, J. L. M.; Sparovek, G.; Meteorol. Z. 2013, 22, 711.

41. Inema - Instituto do Meio Ambiente e Recursos Hídricos, disponível em http://www.inema.ba.gov.br/gestao-2/comites-de-bacias/comites/ cbh-itapicuru, acessado Maio de 2019.
42. Menezes, C. M.; Aguiar, L. G. P. A.; Espinheira, M. J. C. L.; Silva, V. I. S.; Revista Biociências 2009, 15, 44.

43. Shrivastava, A.; Gupta, V. B.; Chron. Young Sci. 2011, 2, 21

44. Guide to quality in analytical chemistry; CITAC, EURACHEM, United Kingdom, 2002.

45. Qian, Y.; Jackson, T.; Standard Operating Procedures from Geochemical and Environmental Research Group, TAMU: College Station, USA, 1997.

46. Godoy, J. M.; Moreira, I.; Wanderley, C.; Simões Filho, F. F.; Mozeto, A. A.; Radiat. Prot. Dosim. 1998, 75, 111.

47. Appleby, P. G.; Oldfield, F.; Catena 1978, 5, 1.

48. Joshi, S. R.; Shukla, B. S. J.; Radioanal. Nucl. Chem. 1991, 148, 73.

49. Garcia, K. S.; Anunciação, D. S.; Jesus, G. M.; Gomes, J. M. P.; Soares, S. A. R.; Garcia, R. J. L. Em Avaliação de ambientes costeiros da região Sul da Bahia: geoquímica, petróleo e sociedade; Celino, J. J., Hadlich, G. M., Queiroz, A. F. S., Oliveira, O. M. C., eds., Edufba: Salvador, 2014, cap. 3.

50. Folk, R. L.; Ward, W. C. J.; Sediment. Petrol. 1957, 27, 3.

51. Santana, J. S.; Monografia de graduação, Universidade Federal da Bahia, Brasil, 2018.

52. Shepard, F. P.; J. Sediment. Petrol. 1954, 24, 151.

53. Pejrup, M.; Em Tide-Influenced Sedimentary Environments and Facies; Boer, P. L.; van Gelder, A.; Nio, S. D., eds.; Reidel: Dordrecht, 1998, cap. 21.

54. Flemming, B. W.; Cont. Shelf Res. 2000, 20, 1125.

55. Ramaswamy, V.; Gaye, B.; Shirodkar, P. V.; Rao, P. S.; Chivas, A. R.; Mar. Chem. 2008, 111, 137.

56. Almeida, A. B., Araújo, F. M., Ribeiro, S. H. S., Pereira, A. M. S., Sampaio, F. J.; Anais do II Congresso sobre Planejamento e Gestão das Zonas Costeiras dos Países de Expressão Portuguesa, Recife, Brasil, 2003.

57. Gearing, P.; Gearing, J. N.; Lytle, T. F.; Lytle, J. S.; Geochim. Cosmoch. Acta 1976, 40, 1005

58. Collister, J. W.; Lichtfouse, E.; Hieshima, G.; Hayes, J. M.; Org. Geochem. 1994, 21, 645.

59. Aboul-Kassim, T. A. T.; Simoneit, B. R. T.; Mar. Chem 1996, 54, 135.

60. Zhang, Z.; Zhao, M.; Eglinton, G.; Lu, H.; Huang, C-Y.; Quat. Sci. Rev. 2006, 25, 575 . 